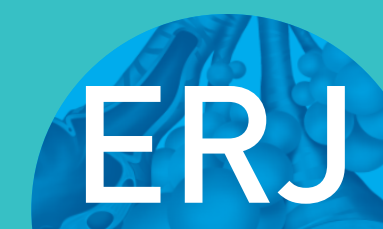

open research
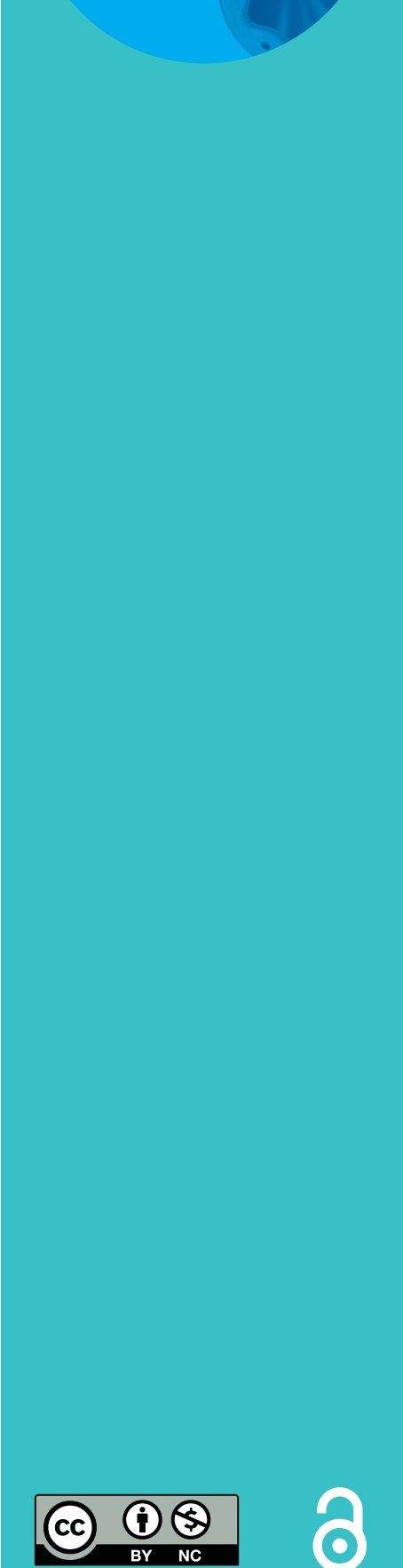

\section{The best of respiratory infections from the 2015 European Respiratory Society International Congress}

\author{
Eva Polverino ${ }^{1,7}$, Graham H. Bothamley ${ }^{2,7}$, Delia Goletti ${ }^{3}$, Jan Heyckendorf ${ }^{4}$, \\ Giovanni Sotgiu ${ }^{5}$ and Stefano Aliberti ${ }^{6}$
}

Affiliations: ${ }^{1}$ Fundació Clinic, Hospital Clinic of Barcelona - Institut d'Investigacions Biomèdiques August Pi i Sunyer, University of Barcelona, Ciber de Enfermedades Respiratorias, Barcelona, Spain. ${ }^{2}$ Dept of Respiratory Medicine, Homerton University Hospital, London, UK. ${ }^{3}$ Translational Research Unit, Dept of Epidemiology and Preclinical Research, National Institute for Infectious Diseases, Rome, Italy. ${ }^{4}$ Division of Clinical Infectious Diseases, German Center for Infection Research, Research Center, Borstel, Germany. ${ }^{5}$ Clinical Epidemiology and Medical Statistics Unit, Dept of Biomedical Sciences, University of Sassari, Medical Education and Professional Development Unit, Sassari, Italy. ${ }^{6}$ Dept of Pathophysiology and Transplantation, University of Milan, Cardio-Thoracic Unit and Cystic Fibrosis Adult Center, Fondazione IRCCS Cà Granda Ospedale Maggiore Policlinico, Milan, Italy. ${ }^{7}$ These authors contributed equally.

Correspondence: Giovanni Sotgiu, Clinical Epidemiology and Medical Statistics Unit, Dept of Biomedical Sciences, University of Sassari, Medical Education and Professional Development Unit, via Padre Manzella, 4, Sassari 07100, Italy. E-mail: gsotgiuauniss.it

ABSTRACT The breadth and quality of scientific presentations on clinical and translational research into respiratory infections at the 2015 European Respiratory Society (ERS) International Congress in Amsterdam, the Netherlands, establishes this area as one of the leadings fields in pulmonology. The host-pathogen relationship in chronic obstructive pulmonary disease, and the impact of comorbidities and chronic treatment on clinical outcomes in patients with pneumonia were studied. Various communications were dedicated to bronchiectasis and, in particular, to different prognostic and clinical aspects of this disease, including chronic infection with Pseudomonas and inhaled antibiotic therapy. Recent data from the World Health Organization showed that Europe has the highest number of multidrug-resistant tuberculosis cases and the poorest countries have the least access to suitable treatments. Latent tuberculosis and different screening programmes were also discussed with particular attention to risk factors such as HIV infection and diabetes. Several biomarkers were proposed to distinguish between active tuberculosis and latent infection. Major treatment trials were discussed (REMOX, RIFQUIN and STREAM). The possibility of once-weekly treatment in the continuation phase (RIAQUIN) was especially exciting. The continuing rise of Mycobacterium abscessus as a significant pathogen was noted. This article reviews some of the best contributions from the Respiratory Infections Assembly to the 2015 ERS International Congress.

@ERSpublications

Members of @ERStalk Assembly 10 discuss the best presentations on respiratory infections at \#ERS2015 http://ow.ly/BVqM3018veM

Received: April 082016 | Accepted after revision: May 272016

Conflict of interest: None declared.

Copyright $\odot$ ERS 2016. This article is open access and distributed under the terms of the Creative Commons Attribution Non-Commercial Licence 4.0. 


\section{Respiratory infections}

A total of 197 abstracts for Group 10.1 of the Respiratory Infections Assembly (Assembly 10) and 171 abstracts for Group 10.2 (Tuberculosis) were presented at the 2015 European Respiratory Society (ERS) International Congress. Communications were allocated into three oral presentation sessions, four poster discussion sessions and six thematic poster sessions. Similarly to previous years, pneumonia has been one of the leading topics, being the main subject of one oral session, one poster discussion and two thematic poster sessions. Interestingly, several communications were also focused on bronchiectasis and infections in other chronic respiratory diseases, such as chronic obstructive pulmonary disease (COPD) and asthma.

The high number of communications on non-cystic fibrosis bronchiectasis characterised different sessions during the congress, including one oral session, mostly dedicated to the analysis of risk factors (i.e. comorbidities, Pseudomonas aeruginosa and thrombocytosis), one poster discussion and one thematic poster session on different clinical and microbiological aspects of the disease. It is worth remarking on the substantial increase in communications regarding bronchiectasis during the 2015 ERS International Congress in comparison to previous congresses, indicating an increasing interest in this orphan disease.

Relevant public attraction was due to symposia, in particular those on the European Registry of Bronchiectasis, the emergence of drug-resistant tuberculosis in Europe and on tuberculosis in the Russian Federation.

\section{Pneumonia}

Different studies of pneumonia were reported mainly focused on factors influencing the host-pathogen interaction. Comorbidities and related medications seem to play a crucial role in determining clinical outcomes of patients with community-acquired pneumonia (CAP). On one hand, a large (11844 cases), retrospective, population-based study by GIL DE Miguel et al. [1] investigated different comorbidities associated with an increased risk of pneumococcal pneumonia in order to provide evidence for the implementation of pneumococcal prevention strategies. Patients with at least one comorbidity showed a higher mortality rate (1.2 versus 0.5 per 1000 inhabitants, $\mathrm{p}<0.01)$, fatality rate $(7.7 \%$ versus $5.7 \%, \mathrm{p}<0.01)$, length of hospital stay (9.6 versus 8.4 days, $\mathrm{p}<0.01)$ and costs $(€ 4935$ versus $€ 4250, \mathrm{p}<0.01)$ than patients without comorbidity. The most relevant comorbidity increasing the risk of pneumococcal pneumonia across all age groups was immunosuppression by accompanying hepatic, cardiovascular, renal or chronic respiratory disease.

However, a Greek multicentre study by Makrodimitri et al. [2] showed that diabetes seems not to be associated per se with greater mortality, although the clinical presentation was more severe due to an increased rate of acute renal failure. The role of comorbidities is also potentially linked to chronic medication, particularly in the elderly and in cases of recurrent pneumonia. In the last decade, inhaled fluticasone and proton pump inhibitors were thought to increase the rate of pneumonia, while statins and angiotensin-converting enzyme (ACE) inhibitors could play a preventive role, being associated with a reduced mortality [3-13]. A Japanese study by IsHifuji et al. [14] on recurrent pneumonia in the elderly showed that age $>75$ years (OR 2.60, 95\% CI 1.06-6.43), previous pneumonia (OR 1.95, 95\% CI 1.35-2.80), chronic pulmonary diseases (OR 1.86, 95\% CI 1.24-2.78) and certain drugs, including hypnotics (OR 2.06, 95\% CI 1.28-3.31) and inhaled steroids (OR 1.78, 95\% CI 1.12-2.84), increase the risk of recurrent pneumonia, while ACE inhibitors seem to be protective (OR 0.22, 95\% CI 0.05-0.91).

A multicentre study from the USA investigated the association between CAP and short- and long-term risk of cardiovascular events and related mortality. During the last year, CoRRALES-MEDINA and co-workers $[15,16]$ described the increased risk of heart failure in the elderly population after pneumonia, although the pathophysiological mechanisms are not completely clear. In line with these findings, SANZ et al. [17] described tachyarrhythmia in one third of 2123 CAP patients and a clear association of pneumococcal aetiology with poor outcomes (particularly with tachyarrhythmia). Further investigation is needed to understand better the pathophysiology of this association in order to design future strategies to reduce the cardiovascular mortality after CAP. A new animal (pig) model of pneumococcal infection was presented by Amaro et al. [18], which was awarded as the "Best Abstract on Bacterial Pneumonia" prize. This model potentially represents a great advance in the study of the pathophysiology of severe invasive pneumococcal pneumonia, offering the possibility to assess the dynamics of systemic and local inflammation, host response, and therapeutic efficacy of any novel treatment. In particular, the sequential measurements of pulmonary mechanics, and sampling of respiratory and systemic biofluids and lung tissue will allow a comprehensive assessment of the effects of pneumococcal infection on the lungs.

\section{Respiratory infections in COPD}

The ERS International Congress hosted presentations of various high-quality studies regarding the host-pathogen balance in COPD-related infections. Microbiome analysis, and the correlation between its compositions and different clinical outcomes was approached by different authors, providing a good example of translational research getting closer to clinical practice. The longitudinal study by Dicker et al. [19] on 
exacerbations of COPD noted that the predominant species of microbiome were Streptococcus spp. or Haemophilus spp., the latter being associated with a higher Global Initiative for Chronic Obstructive Lung Disease (GOLD) stage. In addition, the authors observed that a higher GOLD stage was significantly associated with reduced species diversity ( $\mathrm{p}=0.009$ ). In line with those findings, BriLl et al. [20] showed that Haemophilus influenzae dominance could be associated with greater airway inflammation and bacterial burden. Moreover, Rodrigo-Troyano et al. [21] investigated exacerbations of COPD (ECOPD) due to P. aeruginosa in 401patients, distinguishing between antibiotic-susceptible and -resistant strains (in vitro resistance to one or more class of antibiotics). Interestingly, they observed that the isolation of a resistant $P$. aeruginosa was associated with a reduced mortality risk for ECOPD, suggesting that the presence of a chronic airway infection/colonisation and a possible adaptive immune response could modulate clinical outcomes.

Another interesting topic was the influence of inhaled corticosteroids on the susceptibility to viral infections. STAPLES et al. [22] noted that the exposure to inhaled fluticasone might reduce the activation and infection of T-cells and macrophages; these findings could explain less severe outcomes described in patients receiving chronic inhaled steroids (COPD and asthma) during past influenza pandemics. Conversely, XIA et al. [23] described, in an in vitro and in vivo study (mouse model), that infections caused by respiratory syncytial virus and rhinovirus might induce insensitivity to glucocorticosteroids, partially mediated by transforming growth factor (TGF)- $\beta$. This interesting study could explain the pathophysiology of asthma/COPD exacerbations caused by viral infections as well as the potential usefulness of TGF-modifying drugs for prevention/treatment.

\section{Bronchiectasis}

McDonnell et al. [24] investigated the role of comorbidity in clinical outcomes of bronchiectasis and found that there was a significant association between the number of comorbidities and the Bronchiectasis Severity Index (BSI) (low, 2.9; intermediate, 3.8; high, 4.3) [25] ( $\mathrm{p}<0.0001)$. Comorbidities associated with mortality included COPD (OR 3.95, $\mathrm{p}<0.0001$ ), connective tissue disease (OR 3.37, $\mathrm{p}<0.0001$ ), tumours (OR 4.45, $\mathrm{p}<0.0001$ ), metastatic cancers (OR 10.4, $\mathrm{p}<0.0001$ ), pulmonary hypertension ( OR 3.62, $\mathrm{p}=0.04$ ) and iron deficiency-related anaemia (OR 4.85, $\mathrm{p}=0.005$ ). SALEH et al. reported interesting data on cardiovascular comorbidity in patients with bronchiectasis [26]. The analysis of aortic stiffness (pulse-wave velocity), and cardiac and inflammatory biomarkers among 101 patients with stable bronchiectasis seem to confirm the high cardiovascular risk in these patients. This risk was associated with both exacerbations and an impaired lung function. RENTON et al. [27] found that thrombocytosis, a marker of both inflammation and cardiovascular disease, was associated with an increased severity of bronchiectasis, exacerbation frequency, mortality and reduced quality of life.

Numerous communications were also dedicated to nontuberculous mycobacterial (NTM) infections in bronchiectasis. Notable aspects were the breadth of geographical coverage, with researchers from Japan, the UK and India all contributing. Both basic science studies and clinical trials were focused on biomarkers and antibiotics to treat refractory NTM. The prevalence of NTM infections across different areas of the world was a subject of active debate. The symposium on the European registry of bronchiectasis (EMBARC) [28] was one of the most crowded sessions of the Congress, with almost 2000 attendees. An interesting overview was provided by ALIBERTI et al. [29] on the heterogeneity of the disease (clinical presentation, aetiology and research activity) and on two other crucial aspects of the clinical management of the disease: antibiotic therapy (oral and inhaled) and physiotherapy. Finally, preliminary data from almost 1300 patients recruited to the EMBARC registry focused the attention on disease severity, current treatments (particularly for P. aeruginosa) across Europe, the impact of comorbidities (including COPD) and future expectations for the registry.

During the Congress, research priorities in bronchiectasis previously identified through a Delphi process by patients and experts of the EMBARC Roadmap Working Group were shared and discussed during an oral presentation by AliBERTi et al. [30]. Among the 55 research priorities, the following received special attention. 1) What are the causes of bronchiectasis? 2) What are the triggers of an exacerbation? 3) When should airway drainage techniques be started in patients with bronchiectasis, and which is the most effective and pragmatic? 4) What are the risk factors and causes of fast progression and poor outcomes in patients with bronchiectasis?

\section{Immunosuppression and severe pneumonia}

Various communications were dedicated to fungal infection and, in particular, to pulmonary aspergillosis in immunocompromised patients. Interesting data were presented by SUGISAKI et al. [31] on chronic pulmonary aspergillosis (CPA). The authors showed how underlying respiratory disease influences the clinical features and prognosis of patients with CPA. For instance, patients with emphysema and/or interstitial lung diseases show more rapid progression of CPA, demonstrated by a higher rate of antigen positivity and lower antibody 
titres. Lin et al. [32] analysed a series of 22 cases of CPA over a 5-year period, reporting a higher COPD prevalence $(36.4 \%)$ than in previous studies, and suggested a predisposing role for fungal infections even in the absence of immunosuppressive therapy. In order to improve clinical management of CPA and to reduce diagnostic delay, RozALIYANi et al. [33] proposed a diagnostic score based on four variables: value of lung excreted galactomannan, solid-organ malignancy, pulmonary tuberculosis and systemic corticosteroids. This model will require further validation but it could represent a promising tool to reduce infection-related mortality similarly to severity scores used in other respiratory infections, such as the Pneumonia Severity Index, according to FINE et al. [34] and LuNA et al. [35].

A retrospective, matched case-control study by DesseIGNe et al. [36] investigated Pneumocystis jirovecii pneumonia (PCP) in immunosuppressed patients with solid tumours. They found that severe lymphopenia, $>1$ month corticosteroid treatment and thoracic radiotherapy were significant risk factors for the development of PCP.

\section{Translational research in respiratory infections}

A significant number of research projects were presented this year at the Congress, filling a poster discussion session and various thematic poster sessions. Several aspects of the immunological and inflammatory host response to different pathogens were presented. Various communications focused on the host response to bacterial (e.g. H. influenzae, P. aeruginosa and Streptococcus pneumoniae) and viral (e.g. $\mathrm{H}_{1} \mathrm{~N}_{1}$ influenza virus, respiratory syncytial virus and rhinovirus) infections in healthy subjects and in patients with chronic respiratory diseases, such as asthma. These new findings will hopefully contribute to a better understanding of the host-pathogen interaction and identify novel beneficial approaches beyond antibiotic therapy.

\section{Tuberculosis}

\section{Multidrug-resistant tuberculosis}

The symposium "The emergence of drug-resistant tuberculosis in Europe: no cure possible" began with a patient's perspective following successful treatment for multidrug-resistant tuberculosis (MDR-TB) in Romania. Most notable were the adverse events, the duration of treatment, the experience of relapse, and the stigma afforded to the patient by her relatives and social contacts. Dick van Soolingen (Nijmegen, the Netherlands) championed his contention that the Beijing clade is more virulent than other clades of the Mycobacterium tuberculosis complex by referring to a cluster of 470 individuals (384 from Estonia) who had been infected with this multidrug resistance strain [37]. He suggested from the work of ComAs et al. [38] that compensatory changes for the $r p o B$ mutations in other ribosomal polymerases returned protein manufacture to near normal levels. Furthermore, four proteins that distinguish modern from ancient Beijing strains were shown to be Mmp14 (Rv450c), Rv1269c, CobC (Rv2231c) and SseA (Rv3283), together with three efflux pumps, which might affect intrabacillary levels of antibiotics [39]. A lively discussion noted the potential for founder effects, selection bias (only culture-positive samples can be sequenced) and the role of social group characteristics in the spread of this strain. Unpublished data showed that rifampicin per se induced DosR-regulated genes.

Marieke J. van der Werf (European Centre for Disease Prevention and Control, Stockholm, Sweden) discussed the epidemiology of MDR-TB within the European Union, noting that Romania accounted for $25 \%$ of all MDR-TB but had the lowest rate of routine drug sensitivity testing (DST). In contrast, Christoph Lange (Borstel, Germany) used data from the World Health Organization (WHO) European Region to show that Europe accounted for the highest number of cases of and continued increase in MDR-TB of all the WHO regions. The relative costs of treatment depending on drug resistance patterns showed that the poorest countries of Europe and those with the most significant MDR-TB problem had more limited access to suitable drugs and were paying proportionally more for each second-line drug [40]. The mean costs for even the most expensive drugs that can cure drug-resistant tuberculosis compare favourably with chemotherapy for lung cancer and 10 years' palliative treatment for COPD or asthma according to Rutten-van Mölken et al. [41] and BRiggs et al. [42]. The suggestion was made that in those with fluoroquinolone-resistant tuberculosis, as in extensively drug-resistant tuberculosis (XDR-TB) or pre-XDR-TB, that bedaquiline should be used as the replacement drug of choice.

A symposium on tuberculosis in the Russian Federation noted the problems in containing drug resistance. Results of the implementation of routine DST in three oblasts noted a high rate of MDR-TB [43]. Delays in DST were important in the extended use of first-line drugs and, although MDR-TB treatment failure was only significant if the delay was $>24$ months, the cost of further transmission to the community was thought to be high, as proved by BATYRSHINA [45].

\section{Latent tuberculosis infection and screening}

In a Hot Topics session, Giovanni Sotgiu gave an overview of latent tuberculosis infection (LTBI). A survey of national guidelines against the WHO standard for screening for LTBI indicated most compliance 
when screening contacts and before anti-tumour necrosis factor (TNF) use, but poorer compliance for HIV-infected patients, patients on dialysis or undergoing transplantation, and those with silicosis. The UK programme for screening new entrants was presented. ERKENs et al. [45] noted that preventive treatment with 4 months rifampicin had a higher completion rate in the Netherlands than standard 6-9 months isoniazid. Two large studies from South Korea were presented: KIM et al. [46] showed that even in those with a positive QuantiFERON Gold In-Tube test (Qiagen, Hilden, Germany), if the tuberculin (purified protein derivative (PPD)) skin test diameter was $<10 \mathrm{~mm}$, tuberculosis did not develop (0 out of $152 \mathrm{PPD}^{+}$ contacts out of 3088 tested in total); another study by KIM et al. [47] on screening for diabetes mellitus in 4.9 million subjects identified 11699 with tuberculosis but the association showed a stronger link with age as a confounder for both diabetes and tuberculosis. Surprisingly, Jensen et al. [48] showed that sputum screening in high-risk groups in Copenhagen, Denmark, identified 40 cases of tuberculosis, which were then compared to 56 patients detected by passive screening and shown to have fewer smear-negative samples. This contrasted with the UK experience of MidDLETON et al. [49] in 1039 asylum seekers where 333 out of 379 were referred to a tuberculosis clinic with symptoms or a history of tuberculosis contact, of whom just over $10 \%$ required preventive treatment and a further 15 were given a bacille Calmette-Guérin vaccination. In Portugal, according to Sousa et al. [50], a fall in HIV infection was associated with a concurrent fall in tuberculosis, whereas a rise in unemployment was associated with a small increase in tuberculosis. Data from OzGuler et al. [51] on when to restart TNF treatment if tuberculosis developed were incomplete as a new infection was the cause of tuberculosis as confirmed by a difference in strain typing. Another case of tuberculosis developing during TNF treatment for inflammatory bowel disease again suggested that a new infection rather than reactivation was responsible.

\section{Tuberculosis biomarkers}

GARCía et al. [52] reported the mycobactericidal whole-blood activity in genetically related tuberculosis contacts. In the past, this test has been used to monitor clinical outcome of tuberculosis patients as discussed by WaLlis and co-workers [53, 54]. This study used whole blood mycobactericidal activity to evaluate tuberculosis susceptibility in family contacts and found lower antimycobacterial activity than in the general population, suggesting a genetic susceptibility to tuberculosis, although other immunological explanations are possible. Losi et al. [55] reported on the additional benefits of $M$. tuberculosis-specific antigens to improve upon the interferon (IFN)- $\gamma$ release assays. New antigens were identified by this approach to distinguish LTBI from active disease [56-58]. They screened in silico for human leukocyte antigen DR epitopes of M. tuberculosis proteins in 44 clinical strain genomes. Proteins were ranked accordingly to produce an immunogenetic model of tuberculosis susceptibility. Proteins containing T- and B-cell epitopes were selected, and B-cell epitopes produced as mimotope peptides. Among 121 relevant protein orthologues, 22 proteins were $M$. tuberculosis-specific and were used to prepare 167 mimotopes. 116 of these mimotopes presented a significantly higher IFN- $\gamma$ response in those with active tuberculosis. Antibody testing of the mimotopes gave sensitivities ranging from 27 to $100 \%$ (average 66\%) with a specificity of $100 \%$. These are promising tools to distinguish latent from active tuberculosis, and to explore at both the diagnostic and pathogenetic levels.

Petruccioli et al. [59] reported the evaluation of the accuracy of several immune experimental diagnostic tools to distinguish active tuberculosis from latent infection. They combined several experimental immunological approaches based on the detection of IFN- $\gamma /$ TNF- $\alpha$ bifunctional CD $4^{+} \mathrm{T}$ cells [60], on central memory phenotypes of IFN- $\gamma$-specific CD4 ${ }^{+}$T-cells [61] and an assay based on the "CD27 ratio" [62], discussed in detail in another presentation [63]. By logistic regression followed by receiver operating characteristic analysis, the best combination to discriminate active tuberculosis from LTBI was generated by the use of the detection of the central memory $\mathrm{CD} 4^{+} \mathrm{T}$-cells and the $\mathrm{CD} 27$ ratio (area under the curve $0.96,95 \%$ CI $0.87-0.99 ; \mathrm{p}<0.0001$ ). If confirmed in larger numbers, this approach may help to distinguish tuberculosis disease from LTBI.

Wolf et al. [64] reported on a novel biomarker that was more strongly associated with tuberculosis treatment outcome than conventional or genotypic methods. They developed a modified mycobacteriophage with a fluorescent reporter (F2GFP10), which is able to detect metabolically active M. tuberculosis in biological samples such as sputum. They compared the F2GFP10 assay enumerated by flow cytometry to conventional M. tuberculosis culture and GeneXpert MTB/RIF (Cepheid, Sunnyvale, CA, USA) in serial sputum samples from 20 patients with active tuberculosis who were on treatment. The 6-month treatment outcome was defined by standard WHO criteria and suggested that the new test might have greater accuracy in predicting the outcome of treatment.

\section{Short-course chemotherapy for active tuberculosis}

In a Year in Review session, Stephen H. Gillespie (St Andrew's, UK) presented on the REMOX trial [65]. He noted the superior early bactericidal activity and 2-month culture conversion rates in patients treated with moxifloxacin compared to the standard regimen. However, despite this early effectiveness, the 
standard 6-month regimen could not be shortened to 4 months. PHILLIPs et al. [66] considered that the 3 -month culture conversion rate might be a better predictor of outcome than that at 2 months, although in the REMOX trial relapse occurred in those who had a negative sputum culture. Gillespie suggested that one possibility for the ineffectiveness of 4-month regimens was the initial dose of rifampicin [67]. Alternatively, moxifloxacin might have created more persisters, slowly metabolising and using stored lipids as a substrate [68]. Amina Jindani (London, UK) then reviewed other 4-month regimens, noting the high variability of relapse rates ranging from $11 \%$ with 2 SHRZ/2HRZ to $40 \%$ with 2 SHRZ/2H [69]. The RIFAQUIN trial showed similar rates of relapse (17\%) for a 4-month regimen but notably, the 6-month regimen with weekly rifapentin and isoniazid in the continuation phase was as effective as the standard regimen [69]. Christian Lienhardt (Marseille, France) then reiterated these findings, noting the loss of gatifloxacin from the pharmacopoeia due to adverse effects with a brief overview of the OFLOTUB trial [70]. Sarah Meredith (London) gave an update of the current STREAM trials and the reassessment of variants of the Bangladesh regimen in randomised controlled trials [71]. Shorter treatments for drug-sensitive and -resistant tuberculosis against the standard regimens will probably require a better understanding of the persister stage of M. tuberculosis.

Patients with late sputum conversion were described but no poster confirmed adequate treatment adherence in the initial phase of treatment. A poster by BAE et al. [72] showed that those without sputum conversion at 4 months had a history of previous tuberculosis, acquired multidrug resistance having initially been rifampicin sensitive and had just under 70\% treatment success. MejRi et al. [73] showed a link between diagnostic delays and retrospective assessment of pulmonary cavitation. The importance of early second-line drug sensitivity testing for those with rifampicin resistance was emphasised by UTSAV et al. [74]. Systematic management of drug resistance was important in achieving good outcomes [75, 76]. The toxicity of linezolid was confirmed, with almost $80 \%$ experiencing adverse effects [77] and a fifth developed peripheral neuropathy, half of which was irreversible [78]. Nonadherence to treatment for MDR-TB regimens was frequently due to adverse events (81\%) [79]. A high rate of pre-XDR-TB due to fluoroquinolone resistance was noted in Karachi, Pakistan (363 out of 919 patients with MDR-TB) [80], and Kiev, Ukraine [81]. Rates of cure of XDR-TB continue to be similar to those reported with no treatment in the pre-antibiotic era [81]. There were several presentations noting the outpatient management of M/XDR-TB, although all were evaluated in terms of patient treatment outcomes, which were not different [82], rather than transmission of disease. Importantly, nosocomial transmission of MDR-TB occurred in 75\% of 173 patients who developed MDR-TB having been admitted as inpatients with a fully sensitive strain. The conclusion remains that an early finding of rifampicin resistance should be followed up by rapid second-line testing, preferably genotypically, in order to tailor the drug regimen appropriately, and that systems must be in place to ensure adequate infection control.

\section{Treatment regimens and monitoring}

Moxifloxacin is often used to replace isoniazid in treatment regimens and is effective [83]. Some in the audience raised concerns about the development of resistance to fluoroquinolones in potential pre-MDR-TB/XDR-TB and recommended standard regimens using streptomycin or a longer course with REZ. In vitro evidence was presented that mefloquine, in doses currently used for malarial prevention, can kill M. tuberculosis [84]; other possible drugs were the phenothiazines [85] and others, which in in vitro studies showed a significant percentage of strains with a minimum inhibitory concentration (MIC) apparently above the maximal permissible drug concentrations, although with some technical problems in determining the MIC [86]. A cohort study by Nikolayan et al. [87] was used to justify the use of linezolid, bedaquiline and imipenem as treatment for XDR-TB (Armenia/Russia).

New methods of treatment monitoring were discussed. A study by CoRONEL et al. [88] using AERONOSE suggested there might be changes in exhaled breath in the first 2 months of treatment. The use of magnetic resonance imaging for spinal tuberculosis was advocated by McGHEE et al. [89] but the audience agreed that distinguishing bacterial cure from continuing inflammatory responses and bone remodelling made the use of such an expensive test impractical, especially in the absence of evidence from a randomised controlled trial. Whether there might be strain-related problems with treatment success and relapse was considered by HANG et al. [90] but discounted in the absence of clinical data regarding adherence and other patient-related variables. Video directly observed therapy (vDOT) via mobile phones was the subject of a preliminary trial described by FALzon et al. [91] but participants felt that adherence with vDOT was as likely as without and the intervention could not be used in those in whom adherence was a problem, but rather in national programmes where universal directly observed therapy was required.

\section{Nontuberculous mycobacteria}

Suggestions that NTM and tuberculosis could be radiographically distinguished were made by CHU et al. [92]. The serodiagnosis of Mycobacterium avium complex was again promoted by the Japanese progenitors 
of the glycopeptidolipid antigen test [93, 94]. A poster by LeE et al. [95] suggested that CA 19-9 might be a useful marker of treatment response for M. abscessus pulmonary disease. Laboratory isolation of NTMs due to water contamination are common, according to D'AnTonio et al. [96]. The distinction between isolation and NTM disease remains important, especially in the context of HIV co-infection, as pointed out by ВНАт et al. [97].

\section{Perspectives for 2016 and beyond}

A better understanding of host-related risk factors influencing clinical outcomes in CAP is expected in the future, and could lead to the development of preventive and therapeutic strategies in specific subsets of the population. The identification of different clinical phenotypes and specific prognostic factors is also expected for patients with bronchiectasis due to the increasing number of publications on the field. Moreover, new inhaled antibiotics are expected to be introduced for treatment of bronchiectasis and NTM in the future, subject to confirmatory randomised controlled trials. Finally, the growing research on microbiomes in chronic respiratory diseases such as COPD and bronchiectasis will potentially identify specific disease patterns (stable and exacerbations, chronic infection, etc.) and clinical applications of this innovative and interesting microbiological technique.

The 2015 ERS International Congress in Amsterdam highlighted new approaches in immunodiagnostics and biomarkers for tuberculosis with some potential tests, which now need to be evaluated in larger clinical samples. Emerging MDR-TB/XDR-TB remains a major concern of clinicians throughout the European region, and basic DST and genetic tests need to be more readily available to contain this problem. The 4-month trials have highlighted the differences between the actively dividing population treated in the initial intensive phase of tuberculosis treatment and the more slowly dividing populations that are treated in the continuation phase. This is an area ripe for basic research to contribute real clinical advances. We are looking forward to the next ERS International Congress in London in 2016, where such topics will be addressed.

\section{Acknowledgements}

We thank all the chairs and co-chairs of Assembly 10 for the ERS International Congress 2015 and in particular those who contributed to identifying the most relevant communications of this year: Pieter Goeminne, Michael Loebinger, Anthony De Soyza, Ignacio Martin-Loeches, Christian Wejse, John Hurst, Stephen Gillespie, Amina Jindani, Marieke van der Werf, Dick van Soolingen and Christoph Lange.

\section{References}

1 Gil de Miguel A, Gil-Prieto R, varo-Meca A, et al. Risk of hospitalization due to pneumococcal pneumonia in adults in Spain. The CORIENNE study. Eur Respir J 2015; 46: Suppl. 59, PA1852.

2 Makrodimitri S, Kosmidou A, Avgerinou I, et al. Community-acquired pneumonia-Impacts of diabetes mellitus as a comorbidity: A prospective, observational study. Eur Respir J 2015; 46: Suppl. 59, PA1841.

3 Dang TT, Majumdar SR, Marrie TJ, et al. Recurrent pneumonia: a review with focus on clinical epidemiology and modifiable risk factors in elderly patients. Drugs Aging 2015; 32: 13-19.

4 Almirall J, Bolíbar I, Serra-Prat M, et al. Inhaled drugs as risk factors for community-acquired pneumonia. Eur Respir J 2010; 36: 1080-1087.

5 Drummond MB, Dasenbrook EC, Pitz MW, et al. Inhaled corticosteroids in patients with stable chronic obstructive pulmonary disease: a systematic review and meta-analysis. JAMA 2008; 300: 2407-2416.

6 Janda S, Young A, Fitzgerald JM, et al. The effect of statins on mortality from severe infections and sepsis: a systematic review and meta-analysis. J Crit Care 2010; 25: 656.

7 Schlienger RG, Fedson DS, Jick SS, et al. Statins and the risk of pneumonia: a population-based, nested case-control study. Pharmacotherapy 2007; 27: 325-332.

8 Thomsen RW, Riis A, Kornum JB, et al. Preadmission use of statins and outcomes after hospitalization with pneumonia: population-based cohort study of 29,900 patients. Arch Intern Med 2008; 168: 2081-2087.

9 Myles PR, Hubbard RB, Mckeever TM, et al. Risk of community-acquired pneumonia and the use of statins, ace inhibitors and gastric acid suppressants: a population-based case-control study. Pharmacoepidemiol Drug Saf 2009; 18: 269-275.

10 Chastre J, Fagon JY, Bornet-Lesco M, et al. Evaluation of bronchoscopic techniques for the diagnosis of nosocomial pneumonia. Am J Respir Crit Care Med 1995; 152: 231-240.

11 Gulmez SE, Holm A, Frederiksen $\mathrm{H}$, et al. Use of proton pump inhibitors and the risk of community-acquired pneumonia: a population-based case-control study. Arch Intern Med 2007; 167: 950-955.

12 Sarkar M, Hennessy S, Yang YX. Proton-pump inhibitor use and the risk for community-acquired pneumonia. Ann Intern Med 2008; 149: 391-398.

13 Laheij RJ, Sturkenboom MC, Hassing RJ, et al. Risk of community-acquired pneumonia and use of gastric acid-suppressive drugs. JAMA 2004; 292: 1955-1960.

14 Ishifuji T, Sando E, Kaneko N, et al. Medications associated with the incidence of recurrent pneumonia in Japanese elderly population. Eur Respir J 2015; 46: Suppl. 59, PA1838.

15 Corrales-Medina VF, Taljaard M, Yende S, et al. Intermediate and long-term risk of new-onset heart failure after hospitalization for pneumonia in elderly adults. Am Heart J 2015; 170: 306-312.

16 Corrales-Medina VF, Alvarez KN, Weissfeld LA, et al. Association between hospitalization for pneumonia and subsequent risk of cardiovascular disease. JAMA 2015; 313: 264-274.

17 Sanz F, Restrepo M, Fernández-Fabrellas E, et al. Streptococcus pneumoniae infection is an independent cause of cardiac rhythm alteration in community-acquired pneumonia. Eur Respir J 2015; 46: Suppl. 59, OA3245. 
18 Amaro R, Li Bassi G, Aguilera-Xiol E, et al. Characterization of pulmonary function and mechanics in a novel model of pneumococcal pneumonia. Eur Respir J 2015; 46: Suppl. 59, OA3251.

19 Dicker A, Crichton M, Cassidy A, et al. Preliminary data from a longitudinal study of the airway microbiome in COPD. Eur Respir J 2015; 46: Suppl. 59, OA4748.

20 Brill SE, James P, Cookson WOC, et al. Relationship between COPD characteristics and airway bacterial load measured using 16S qPCR. Eur Respir J 2015; 46: Suppl. 59, PA3019.

21 Rodrigo-Troyano A, Suárez-Cuartin G, Peiro M, et al. Acute exacerbations of COPD due to pseudomonas aeruginosa: impact of antimicrobial resistance. Eur Respir J 2015; 46: Suppl. 59, OA4753.

22 Staples KJ, McKendry RT, Spalluto CM, et al. Evidence for cell mediated immune dysfunction in the COPD lung The role of cytotoxic CD4+ T cells. Eur Respir J 2015; 46: Suppl. 59, PA2603.

23 Xia YC, Langenbach S, Radwan A, et al. Respiratory syncytial virus induces glucocorticoid insensitivity. Eur Respir J 2015; 46: Suppl. 59, OA4751.

24 McDonnell M, Fardon T, Rutherford R, et al. Impact of co-morbidities on disease severity and risk of mortality in non-cystic fibrosis bronchiectasis. Eur Respir J 2015; 46: Suppl. 59, OA467.

25 Chalmers JD, Goeminne P, Aliberti S, et al. The Bronchiectasis Severity Index. An international derivation and validation study. Am J Respir Crit Care Med 2014; 189: 576-585.

26 Saleh A, Kwok B, Brown J, et al. Determinants and assessment of excess cardiovascular risk in bronchiectasis Eur Respir J 2015; 46: Suppl. 59, OA468.

27 Renton D, Hill J, Abo-Leyeh H, et al. Thrombocytosis is associated with disease severity and outcomes in stable bronchiectasis. Eur Respir J 2015; 46: Suppl. 59, OA469.

28 Chalmers JD, Aliberti S, Polverino E, et al. The EMBARC European Bronchiectasis Registry: protocol for an international observational study. ERJ Open Res 2016; 2: 00081-2015.

29 Aliberti S, Lonni S, Dore S, et al. Clinical phenotypes in adult patients with bronchiectasis. Eur Respir J 2016; 47: $1113-1122$.

30 Aliberti S, Masefield S, Polverino E, et al. Research priorities in bronchiectasis: a consensus statement from the EMBARC Clinical Research Collaboration. Eur Respir J 2016 [in press DOI: 10.1183/13993003.01888-2015].

31 Sugisaki M, Hagiwara E, Ito $\mathrm{H}$, et al. Impact of underlying respiratory diseases on clinical features and prognosis of chronic pulmonary aspergillosis. Eur Respir J 2015; 46: Suppl. 59, PA2652.

32 Lin SJ, Schranz J, Teutsch SM. Aspergillosis case-fatality rate: systematic review of the literature. Clin Infect Dis 2001; 32: 358-366.

33 Rozaliyani A, Jusuf A, Prihartono J, et al. Model of diagnosis scoring, clinical and molecular profile of invasive pulmonary aspergillosis (IPA) in ICU patients. Eur Respir J 2015; 46: Suppl. 59, PA574.

34 Fine MJ, Auble TE, Yealy DM, et al. A prediction rule to identify low-risk patients with community-acquired pneumonia. N Engl J Med 1997; 336: 243-250.

35 Luna CM, Blanzaco D, Niederman MS, et al. Resolution of ventilator-associated pneumonia: prospective evaluation of the clinical pulmonary infection score as an early clinical predictor of outcome. Crit Care Med 2003 31: $676-682$

36 Desseigne $\mathrm{M}$, Avrillon $\mathrm{V}$, Bienvenu AL, et al. Risk factors of pneumocytis pneumoniae in solid tumours: A case-control study. Eur Respir J 2015; 46: Suppl. 59, PA576.

37 Merker M, Blin C, Mona S, et al. Evolutionary history and global spread of the Mycobacterium tuberculosis Beijing lineage. Nat Genet 2015; 47: 242-249.

38 Comas I, Borrell S, Roetzer A, et al. Whole-genome sequencing of rifampicin-resistant Mycobacterium tuberculosis strains identifies compensatory mutations in RNA polymerase genes. Nat Genet 2012; 44: 106-110.

39 de Keijzer J, de Haas PE, de Ru AH, et al. Disclosure of selective advantages in the "modern" sublineage of the Mycobacterium tuberculosis Beijing genotype family by quantitative proteomics. Mol Cell Proteomics 2014; 13: $2632-2645$.

40 Günther G, Gomez GB, Lange C, et al. Availability, price and affordability of anti-tuberculosis drugs in Europe: a TBNET survey. Eur Respir J 2015; 45: 1081-1088.

41 Rutten-van Mölken MPMH, Goossens LMA. Cost effectiveness of pharmacological maintenance treatment for chronic obstructive pulmonary disease: a review of the evidence and methodological issues. Pharmacoeconomics 2012; 30: 271-302

42 Briggs A, Glick H, Lozano-Ortega G, et al. The TORCH (towards a revolution in COPD health) study: salmeterol/ fluticasone propionate improves quality adjusted survival over three years. Thorax 2006; 61: Suppl. 2, ii39.

43 Testov V, Puzanov V, Yakimova M, et al. Drug resistance among new TB cases in some Russian regions. Eur Respir J 2015; 46: Suppl. 59, PA2717.

44 Batyrshina Y. Impact of pre-treatment duration by first line drugs on treatment outcomes of patients with MDR-TB. Eur Respir J 2015; 46: Suppl. 59, PA3337.

45 Erkens C, Slump E, Verhagen M, et al. The effectiveness of programmatic management of latent tuberculosis infection in the Netherlands. Eu Respir J 2015; 46: Suppl. 59, OA1446.

46 Kim HJ, Oh SY, Lee JB, et al. Tuberculin skin testing to the IGRA positive contacts of infectious tuberculosis cases for the treatment of latent tuberculosis infection. Eur Respir J 2015; 46: Suppl. 59, OA1447.

47 Kim HJ, Cho EH, Lee EP, et al. Effect of blood glucose level on the development of tuberculosis based on national health screening program. Eur Respir J 2015; 46: Suppl. 59, OA1453.

48 Jensen SG, Olsen NW, Seersholm N, et al. Infectiousness of tuberculosis patients identified through spot sputum screening compared to patients identified through passive case finding-comparing sputum smear microscopy results. Eur Respir J 2015; 46: Suppl. 59, OA1448.

49 Middleton A, Howie J, Clarke T, et al. Can asylum seekers be traced! Eur Respir J 2015; 46: Suppl. 59, OA1449.

50 Sousa P, Oliveira A, Gaio R, et al. Longitudinal clustering of tuberculosis incidence in Portugal from 2002 to 2012. Eur Respir J 2015; 46: Suppl. 59, OA1450.

51 Ozguler Y, Hatemi G, Ugurlu S, et al. Restarting biologic agents in rheumatology patients who developed tuberculosis under TNFa-inhibitor treatment. 2015; 46: Suppl. 59, OA1451.

52 García JJ, García SR, García SG, et al. Mycobactericide activity in genetically related tuberculosis contacts Eur Respir J 2015; 46: Suppl. 59, OA3484. 
53 Wallis RS, Vinhas SA, Johnson JL, et al. Whole blood bactericidal activity during treatment of pulmonary tuberculosis. J Infect Dis 2003; 187: 270-278.

54 Wallis RS, Dawson R, Friedrich SO, et al. Mycobactericidal activity of sutezolid (PNU-100480) in sputum (EBA) and blood (WBA) of patients with pulmonary tuberculosis. Plos One 2014; 9: e94462.

55 Losi M, Knights AJ, Mariani F, et al. QuantiFERON-TB performance enhanced by novel Mycobacterium tuberculosis-specific antigens. Eur Respir J 2016; 47: 660-664.

56 Nikolova M, Markova R, Drenska R, et al. Antigen-specific CD4- and CD8-positive signatures in different phases of Mycobacterium tuberculosis infection. Diagn Microbiol Infect Dis 2013; 75: 277-281.

57 Grifoni A, Montesano C, Patronov A, et al. Immunoinformatic docking approach for the analysis of KIR3DL1/HLA-B interaction. Biomed Res Int 2013; 2013: 283805.

58 Giacò L, Amicosante M, Fraziano M, et al. B-Pred, a structure based B-cell epitopes prediction server. Adv Appl Bioinform Chem 2012; 5: 11-21.

59 Petruccioli E, Petrone L, Chiacchio T, et al. Evaluation of the accuracy of several immune experimental diagnostic tools to distinguish active tuberculosis from latent infection. Eur Respir J 2015; 46: Suppl. 59, OA3486.

60 Petruccioli E, Petrone L, Vanini V, et al. IFN gamma/TNF alpha specific-cells and effector memory phenotype associate with active tuberculosis. J Infect 2013; 66: 475-486.

61 Chiacchio T, Petruccioli E, Vanini V, et al. Polyfunctional T-cells and effector memory phenotype are associated with active TB in HIV-infected patients. J Infect 2014; 69: 533-545.

62 Petruccioli E, Petrone L, Vanini V, et al. Assessment of CD27 expression as a tool for active and latent tuberculosis diagnosis. J Infect 2015; 71: 526-533.

63 Petruccioli E, Petrone L, Vanini V, et al. CD27 expression as a new tool to distinguish active tuberculosis from latent infection. Eur Respir J 2015; 46: Suppl. 59, OA3487.

64 Wolf A, O’Donnell M, Jain P, et al. Novel biomarker more strongly associated with tuberculosis treatment outcome than conventional or genotypic methods. Eur Respir J 2015; 46: Suppl. 59, OA3489.

65 Gillespie SH, Crook AM, Mchugh TD, et al. Four-month moxifloxacin-based regimens for drug-sensitive tuberculosis. N Engl J Med 2014; 371: 1577-1587.

66 Phillips PPJ, Fielding K, Nunn AJ. An evaluation of culture results during treatment for tuberculosis as surrogate endpoints for treatment failure and relapse. PLoS One 2013; 8: e63840.

67 Boeree MJ, Diacon AH, Dawson R, et al. A dose-ranging trial to optimize the dose of rifampin in the treatment of tuberculosis. Am J Respir Crit Care Med 2015; 191: 1058-1065.

68 Bothamley GH. Fluoroquinolone preventive therapy after exposure to multidrug-resistant tuberculosis? Hopes and fears. Am J Respir Crit Care Med 2015; 192: 131-132.

69 Jindani A, Harrison TS, Nunn AJ, et al. High-dose rifapentine with moxifloxacin for pulmonary tuberculosis N Engl J Med 2014; 371: 1599-1608.

70 Merle CS, Fielding K, Sow OB, et al. A four-month gatifloxacin-containing regimen for treating tuberculosis. N Engl J Med 2014; 371: 1588-1598.

71 Nunn AJ, Rusen ID, Van Deun A, et al. Evaluation of a standardized treatment regimen of anti-tuberculosis drugs for patients with multi-drug-resistant tuberculosis (STREAM): study protocol for a randomized controlled trial. Trials 2014; 15: 353.

72 Bae S, Yeo Y, Jo K, et al. Clinical characteristics and treatment outcomes of patients with pulmonary tuberculosis with delayed conversion over 4 months. Eur Respir J 2015; 46: Suppl. 59, PA2694.

73 Mejri I, Ben Saad S, Daghfous H, et al. Factors influencing sputum smear conversion delay in tuberculosis Eur Respir J 2015; 46: Suppl. 59, PA2695.

74 Utsav K, Solanki RN. Case series of 83 XDR-TB patients in an urban hospital of Gujarat, India; an interim analysis. Eur Respir J 2015; 46: Suppl. 59, PA2697.

75 Oliveira O, Silva A, Antunes A, et al. Centralization of M/XDR-TB care in clinical reference centres in Portugal. Experience of a regional reference centre. Eur Respir J 2015; 46: Suppl. 59, PA2700.

76 Rao D, Rao N, Baig S, et al. Treatment outcome of MDR pulmonary tuberculosis in a tertiary care hospital of Karachi, Pakistan. Eur Respir J 2015; 46: Suppl. 59, PA2701.

77 Skrahina A, Hurevich H, Falzon D, et al. Active pharmacovigilance for multidrug-resistant tuberculosis patients treated with Linezolid, Belarus -- 2014-2015. Eur Respir J 2015; 46: Suppl. 59, PA2704

78 Tiwari M, Patel M, Shamaliya K. Peripheral neuropathy in XDR-TB patients on second line anti-tubercular therapy. Eur Respir J 2015; 46: Suppl. 59, PA2710.

79 Snène H, Daghfous H, Ben Saad S, et al. Failure predictors when treating MDR-TB. Eur Respir J 2015; 46: Suppl. 59, PA2699.

80 Rao N, Baig S, Hussain N, et al. Prevalence of pre-XDR-TB, XDR-TB among MDR-TB Patients registered at Ojha Institute of Chest Diseases, Karachi. Eur Respir J 2015; 46: Suppl. 59, PA2715.

81 Cherenko S, Litvinenko N. Prevalence and trend of ofloxacin-resistance among MDR-TB including XDR-TB cases in Ukraine. Eur Respir J 2015; 46: Suppl. 59, PA2721.

82 Taran D, Golubchikov P. MDR TB treatment results of GFATM program implementation in Tomsk, Russia. Eur Respir J 2015; 46: Suppl. 59, PA2735.

83 Kuzhko M, Procyk L, Grechanyk L, et al. Efficacy of moxifloxacin in TB resistant to isoniazid. Eur Respir J 2015; 46: Suppl. 59, PA3328.

84 Krieger D, Vesenbeckh S, Schönfeld N, et al. Minimal inhibitory concentrations (MICs) of mefloquine: in vitro activity against MDR- and nonMDR-Tb strains. Eur Respir J 2015; 46: Suppl. 59, PA3327.

85 Vesenbeckh S, Krieger D, Bettermann G, et al. Neuroleptic drugs as potential adjuvants in the treatment of MDR-TB: Minimal inhibitory concentrations (MICs) of different phenothiazines against M. tuberculosis. Eur Respir J 2015; 46: Suppl. 59, PA3329.

86 Popov S, Kuzmin A, Sabgayda T, et al. Minimum inhibitory concentrations (MIC) determination of TB drugs and broad-spectrum antibiotics in M.tuberculosis with M/X/TDR. Eur Respir J 2015; 46: Suppl. 59, PA3331.

87 Nikolayan LT, Yeghiazaryan LM, Hayrapetyan AO, et al. Administration of linezolid, bedaquiline and imipenem in treatment of patients with extensively drug-resistant tuberculosis. Eur Respir J 2015; 46: Suppl. 59, PA3336.

88 Coronel R, Gonzalez S, Magis-Escurra C. Electronic nose as a surrogate marker of response to treatment of pulmonary tuberculosis (ELNOSUR study). Eur Respir J 2015; 46: Suppl. 59, PA3340. 
McGhee E, Chengappa K, Breen R, et al. Should MRI be used to determine the duration of treatment in spinal tuberculosis? Eur Respir J 2015; 46: Suppl. 59, PA3342.

90 Hang NTL, Maeda S, Wada T, et al. Influence of mycobacterium tuberculosis strains on recurrence of tuberculosis in Hanoi, Vietnam. Eur Respir J 2015; 46: Suppl. 59, PA3344.

91 Falzon D, Raviglione M, Bettcher D, et al. Joint WHO/ERS initiative on e-/m-Health for TB and tobacco control. Eur Respir J 2015; 46: Suppl. 59, PA1536.

92 Chu H, Li B, Zhao L, et al. Chest imaging comparison between non-tuberculous and tuberculosis mycobacteria in sputum acid fast bacilli smear-positive patients. Eur Respir J 2015; 46: Suppl. 59, PA2674.

93 Kobashi Y, Mouri K, Kato S, et al. Clinical evaluation of glycopeptidolipid core antigen IgA antibodies for serodiagnosis of mycobacterium avium complex disease. Eur Respir J 2015; 46: Suppl. 59, PA2675.

94 Kishino Y, Ohnishi T, Kusumoto S, et al. Retrospective investigation of the usefulness of GPL-core antibody for diagnosing MAC-PD in patients who had undergone bronchoscopic examination. Eur Respir J 2015; 46: Suppl. 59, PA2676.

95 Lee E, Hong JY, Kang YA, et al. The change in serum Ca 19-9 levels according to treatment responses in pulmonary nontuberculous mycobacterial disease. Eur Respir J 2015; 46: Suppl. 59, PA2677.

96 D’Antonio S, Orazi D, Magini E, et al. Non tuberculous mycobacteria (NTM) contamination in a hospital water supply network: Association with pulmonary infection in respiratory wards. Eur Respir J 2015; 46: Suppl. 59, PA2682.

97 Bhat J, Rajhan A, Agarwal S, et al. Non-tuberculous mycobacterium incidence \& treatment rates in university hospitals of North Midlands (UK). Eur Respir J 2015; 46: Suppl. 59, PA2683. 